\title{
THE FORMAL COMPLETION OF THE NÉRON MODEL OF $J_{0}(p)$
}

\author{
ENRIC NART*
}

Abstract

For any prime number $p>3$ we compute the formal completion of the Neron model of $J_{0}(p)$ in terms of the action of the Hecke algebra on the $\mathbb{Z}$ module of all cusp forrus (of weight 2 with respect to $\Gamma_{0}(p)$ ) with integral Fourier developement at infinity.

Let $p$ be a prime number greater than three. Let $\mathcal{J}_{\mid \mathbb{Z}}$ be the Néron model of the jacobian $J_{0}(p)_{\mid \notin}$ of the modular curve $X_{0}(p)_{\mid Q}$. In a joint work with Deninger we proved that the formal completion of $\mathcal{J}$ along the zero section is determined by the relative $L$-series of $J_{0}(p)$ with respect to $\mathbb{\nabla} \otimes Q$, where $\mathbb{T}$ is the Hecke algebra [2]. In fact, we explained how to construct a formal group law for $\mathcal{J}^{\wedge}$ from a formal Dirichlet series made up with the integral matrices refecting the action of the Hecke operators on the Lie algebra of $\mathcal{J}$.

In this note we show that such a formal group law can also be constructed with the integral matrices reflecting the action of $\mathbb{U}$ on the $\mathbb{Z}$-module $S_{2}\left(\Gamma_{0}(p), \mathbb{Z}\right)$ of all cusp forms (of weight 2 , with respect to $\Gamma_{0}(p)$ ) with integral Fourier developement at infinity. We obtain in this way an effective result since, with the aid of a computer, it is possible to find explicit $\mathbb{Z}$-basis of $S_{2}\left(\Gamma_{0}(p), \mathbb{Z}\right)$ and to compute the action of the Hecke algebra.

Acknowledgements. This question was raised in a conversation with Christopher Deninger. I thank him for this and the IHES at Bures-sur-Yvette for hospitality.

Let $g$ be the dimension of $J_{0}(p)$. Our aim is to prove the following theorem:

*partially supported by grant PB89-0215-0 from CICYT 
Theorem. Let $U_{p} \in M_{g}(\mathbb{Z})$ and $T_{l} \in M_{g}(\mathbb{Z})$, for all primes $l \neq p$, be the matrices of the Atkin-Lehner operator and the Hecke operators, with respect to any $\mathbb{Z}$-basis of $S_{2}\left(\Gamma_{0}(p), \mathbb{Z}\right)$. Since these matrices commute, the formal Dirichlet series:

$$
\sum_{n=1}^{\infty} A_{n} \cdot n^{-s}=\left(I_{g}-U_{p} \cdot p^{-s}\right)^{-1} \cdot \prod_{l}\left(I_{g}-T_{l} \cdot p^{-s}+I_{g} \cdot p^{1-2 s}\right)^{-1},
$$

is well-defined and $A_{n} \in M_{g}(\mathbb{Z})$ for all $n$. Let $L(X, Y)$ be the $g$-dimensional formal group law with logarithm:

$$
f(x)=\sum_{n=1}^{\infty} \frac{1}{n} A_{n} X^{n} \in \mathbb{Q}\left[X_{1}, \ldots, X_{g}\right]^{9},
$$

where $X^{n}$ is the notation for $\left(X_{1}^{n}, \ldots, X_{g}^{n}\right)^{t}$. Then, $L(X, Y)$ is defined over $\mathbb{Z}$ and it is isomorphic to the formal completion of $\mathcal{J}$ along the zero section.

Honda [4] proved an analogous result for Shimura curves, but, a finite (fairly big) set of primes had to be left aside. In fact, our proof follows the same pattern, but we have at our disposal deep results of Deligne-Rapoport [1], Deligne [5, thm. A.1] (which was implicitely used in [2]) and Mazur [5, II, sections 3 and 6], which allow us to deal with the bad primes.

After [2], in order to prove the theorem it is sufficient to show that Lie $(\mathcal{J})$ and $S_{2}\left(\Gamma_{0}(p), \mathbb{Z}\right)$ are isomorphic as $\mathbb{T}$-modules. To this aim is devoted the rest of the papcr. The proof consists on adding some details (checking of some compatibilities, essentially) to certain results of Mazur.

For any integer $N \geq 5$, let $M_{0}(N)$ be the curve over $\mathbb{Z}$ representing the fine moduli stack classifying generalized elliptic curves over $\mathbb{Z}[1 / N]$ with a cyclic subgroup of order $N$. Let $X_{0}(N) \stackrel{i}{\longrightarrow} M_{0}(N)$ be its minimal regular resolution. These two curves become isomorphic over $\mathbb{Z}[1 / N]$.

The Atkin involution $\mathrm{w}=\mathrm{w}_{N}$ extends to an involution of $M_{0}(N)$ [1, IV, Prop. 3.19] and by minimality, to an involution of $X_{0}(N)$ commuting with $i$. Hence, w acts on $H^{1}\left(X_{0}(N), \mathcal{O}\right)$ and on $H^{1}\left(M_{0}(N), \mathcal{O}\right)$ in a compatible way. That is, we have a commutative diagram:

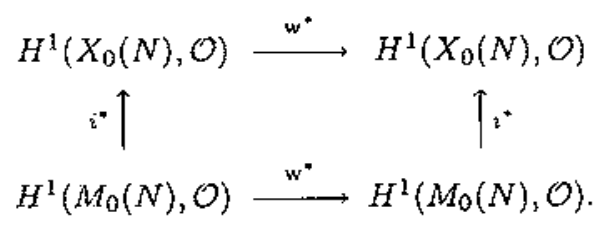

Now, let $l$ be $z$ prime different from $p$ and consider the finite morphism [5, II, section 6]:

$$
\text { c. } M_{0}(p l) \longrightarrow M_{0}(p),\left(E,\left(H_{l}, H_{p}\right)\right) \longrightarrow\left(E, H_{p}\right)
$$


Here $\left(H_{i}, H_{p}\right)$ denotes a cyclic subgroup of $E$ of order $p l$ (canonically) decomposed as a product of its $p$-primary and $l$-primary parts. By minimality $c$ raises to a finite morphism between the regular resolutions fitting into a commutative diagram:

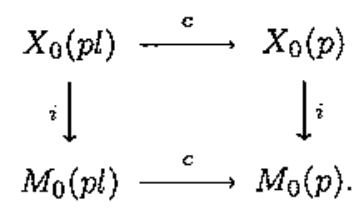

Let us denote $X=X_{0}(p), X^{\prime}=X_{0}(p l), M=M_{0}(p), M^{\prime}=M_{0}(p l)$. The morphism $c: X^{\prime} \longrightarrow X$ induces covariant and contravariant homomorphisns:

$$
\mathrm{Pic}_{X^{\prime} / \mathbb{Z}}^{0} \underset{c_{*}}{\stackrel{c^{*}}{\leftrightarrows}} \mathrm{Pic}_{X / \mathbb{Z}}^{0}
$$

At the level of invertible sheafs, $c^{*}$ is the usual homomorphism and $c_{*}$ is the norm-homomorphism defined by Grothendieck $[3,6.5]$. Via the canonical identification of $H^{1}(X, \mathcal{O})$ with the tangent space of $\mathrm{Pic}_{X / \mathbb{Z}}^{0}$ at the zero-section, $c_{*}$ and $c^{*}$ induce homomorphisms:

$$
H^{1}\left(X^{\prime}, \mathcal{O}\right) \underset{c .}{\stackrel{c^{*}}{\leftrightarrows}} H^{1}(X, \mathcal{O})
$$

$c^{*}$ is the natural homomorphism induced by $\mathrm{O}_{X} \longrightarrow c_{*} \mathcal{O}_{X^{\prime}}$ and the homomorphism $H^{1}\left(X, c_{n} \mathcal{O}_{X^{\prime}}\right) \longrightarrow H^{ \pm}\left(X^{\prime}, \mathcal{O}_{X^{\prime}}\right)$ given by the Leray spectral sequence; whereas $c_{*}$ is the trace-homomorphism defined in terms of Cech cocycles by:

$$
c_{*}\left(f_{\alpha \beta}\right)=\operatorname{Tr}_{X^{\prime} \mid X}\left(f_{\alpha \beta}\right),
$$

for any affine open covering: $X^{\prime}=\cup_{\alpha} c^{-1}\left(U_{\alpha}\right)$, for $U_{\alpha}$ an affine open covering of $X$. This trace is well-defined since $\Gamma\left(c^{-1} U_{\alpha}, \mathcal{O}_{X^{\prime}}\right)$ is a finite $\Gamma\left(U_{\alpha}, \mathcal{O}_{X}\right)$-module.

In fact, the identification of $H^{l}(X, O)$ with the tangent space of $\mathrm{Pic}_{X / \mathbb{Z}}^{0}$ can be realized through the exact sequence:

$$
0 \longrightarrow H^{1}(X, \mathcal{O}) \stackrel{\exp }{\longrightarrow} H^{1}\left(X \otimes \mathbb{z}[\varepsilon], \mathcal{O}^{*}\right) \longrightarrow H^{1}\left(X, \mathcal{O}^{*}\right),
$$

where $\mathbb{Z}[\varepsilon]$ is the ring of dual numbers and $\exp (f)=1+f \varepsilon$. The above description of the action of $c^{*}$ and $c_{*}$ can be easily deduced from this sequence, working with Cech cocycles and having in mind that $1+\operatorname{Tr}_{X^{\prime} / X}(f) \varepsilon$ is the norm of $1+f \varepsilon$. 
By Grothendieck duality we obtain homornorphisms:

$$
H^{0}\left(X^{\prime}, \Omega_{X^{\prime}}\right) \underset{\text { c. }}{\stackrel{c^{*}}{\leftrightarrows}} H^{0}\left(X, \Omega_{X}\right)
$$

where $\Omega_{X}$ is the dualizing sheaf, that is, the sheaf of regular differentials, which is defined as the only non-vanishing homology group (in degree -1) of the complex $R \pi{ }^{\prime} \mathcal{O}_{\text {Spec }}$, where $\pi$ is the structural morphism of $X$.

We need to check the compatibility of these homomorphisms $c_{*}, c^{*}$ with the analogous homomorphisms defined by Mazur at the level of the curves $M_{0}(N)$ $\left[5\right.$, page 88], which we denote by $\left(c^{*}\right)_{M},\left(c_{*}\right)_{M}$. More precisely, we need the following diagrams to commute:

$$
\begin{aligned}
& H^{1}\left(X^{\prime}, \mathcal{O}\right) \stackrel{c^{*}}{\longleftarrow} H^{1}(X, \mathcal{O}) \\
& i^{*} \uparrow \quad i^{*} \\
& H^{1}\left(M^{\prime}, \mathcal{O}\right) \stackrel{\left(c^{*}\right)_{M}}{\longleftarrow} H^{1}(M, \mathcal{O}) \\
& H^{0}\left(X^{\prime}, \Omega\right) \stackrel{\epsilon^{*}}{\longleftarrow} H^{0}(X, \Omega) \\
& \text { i. } \quad \text { \. } \\
& H^{0}\left(M^{\prime}, \Omega\right) \stackrel{\left(c^{*}\right)_{M}}{\longleftarrow} H^{0}(M, \Omega) \text {, }
\end{aligned}
$$

where $i_{*}$ is defined from $i^{*}$ by duality. Now, diagram (3) commutes since it is obtained from (2) by taking everywhere the natural homomorphisms induced by $i$ and $c$. Since the $\mathbb{Z}$-modules involved are free [5, II, Lemma 3.3 and (3.2)] it is sufficient to check the commutativity of diagram (4) after tensoring with $Q$. Then, the commutativity amounts to the fact that the natural homomorphism: $H^{0}\left(X_{\mathbb{Q}}^{\prime}, \Omega^{1}\right) \stackrel{c^{*}}{\longleftarrow} H^{0}\left(X_{\mathbb{Q}}, \Omega^{1}\right)$ is dual to the trace-homomorphism, $\operatorname{Tr}_{X^{\prime} / X}: H^{1}\left(X_{\mathbb{Q}}^{\prime}, \mathcal{O}\right) \longrightarrow H^{1}\left(X_{\mathbb{Q}}, \mathcal{O}\right)$, under Serre duality, and this is a consequence of the classical trace-formula [7, page 32].

We are ready to analize the action of the Hecke algebra. The Hecke algebra $\sigma$ is the subalgebra of End $\left(J_{0}(p)\right)$ generated by all the operators $T_{l}$ and the Atkin involution $w$. The Hecke operator $T_{l}$ is, by definition, the endomorphism of $J_{0}(p)$ induced by correspondence on $X_{0}(p)_{1}$ determined by the morphisms:

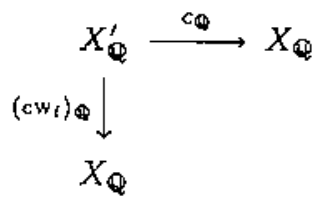


To be more precise, $T_{i}$ is the composition of the two homomorphisms:

$$
T_{i}: J_{0}(p) \stackrel{\left(c w_{i}\right) \dot{\phi}}{\longrightarrow} J_{0}(p l) \stackrel{(c \infty)}{\longrightarrow} J_{0}(p)
$$

induced by $c_{Q}$ and $\left(\mathrm{Cw}_{l}\right)_{Q}$ on $\mathrm{Pic}_{X_{0}(N) / Q}^{0}=J_{0}(N)$, for $N=p, p l$. By the universal property of the Néron model, $T_{l}$ operates on $\mathcal{J}$ and on its connected component as:

$$
T_{l}: \mathcal{J}^{0} \stackrel{\left(c w_{l}\right)_{z}^{*}}{\longrightarrow}\left(\mathcal{J}^{\prime}\right)^{0} \stackrel{\left(c_{*}\right)_{z}}{\longrightarrow} J^{0}
$$

where $\left(\mathcal{J}^{\prime}\right)^{0}$ is the connected component of the Néron model of $J_{0}(p l)$. By a theorem of Raynaud $[6,8.1 .4]$, the connected component of the Nerron model of $J_{0}(N)$ represents the functor Pic $_{X_{0}(N) / \mathbb{Z}}^{0}$. Hence the homomorphisms:

$$
\operatorname{Pic}_{X^{+} / \mathbb{Z}}^{0} \underset{c_{0}}{\stackrel{\left(\mathrm{cw}_{l}\right)^{\bullet}}{\leftrightarrow}} \mathrm{Pic}_{X / \mathbb{Z}}^{0}
$$

induced by the finite morphisms $X^{\prime} \stackrel{c_{c} c w_{t}}{\longrightarrow} X$, coincide with $\left(c w_{l}\right)_{\mathbb{Z}}^{*},\left(c_{*}\right)_{\mathbb{Z}}$, since they induce the same homomorphism on the generic fiber. Thus, $T_{l}$ acts on $H^{1}(X, O)$ and (by duality) on $H^{0}(X, \Omega)$. We have a commutative diagram:

$$
\begin{array}{cccc}
T_{l}: H^{1}(X, \mathcal{O}) & \stackrel{\left(\mathrm{cw}_{l}\right)^{*}}{\longrightarrow} H^{1}\left(X^{\prime}, \mathcal{O}\right) & \stackrel{c_{*}}{\longrightarrow} H^{1}(X, \mathcal{O}) \\
i^{\bullet} \uparrow & i^{\bullet} \uparrow & & \uparrow^{*} \\
H^{1}(M, \mathcal{O}) & \stackrel{\left(c w_{l}\right)_{M}^{*}}{\longrightarrow} H^{1}\left(M^{\prime}, \mathcal{O}\right) \stackrel{\left(c_{*}\right)_{M}}{\longrightarrow} H^{1}(M, \mathcal{O})
\end{array}
$$

The left-hand square is diagram (3) for $\mathrm{cw}_{l}$ and the right-hand square is the dual of diagram (4). Mazur shows that $i^{*}: H^{1}(M, \mathcal{O}) \longrightarrow H^{1}(X, \mathcal{O})$ is an isomorphism [5, II, Prop. 3.4]; hence, through this isomorphism we obtain (by (I) and (5)) the same structure of $\sigma$-module on $H^{1}(M, O)$ as the one taken ty definition by Mazur. That is, we have isomorphisms as $\overline{\text { 耳-modules: }}$

$$
H^{1}\left(X, \mathcal{O} \cong H^{1}(M, \mathcal{O}), \quad H^{0}(X, \Omega) \cong H^{0}(M, \Omega)\right.
$$

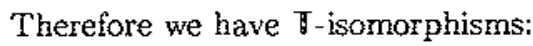

$$
\operatorname{Lie}\left(J^{0}\right) \cong T_{0}\left(J^{0}\right)^{\wedge} \cong H^{1}(X, \mathcal{O})^{\wedge} \cong H^{0}(X, \Omega) \cong H^{0}(M, \Omega) \text {, }
$$

and this last group is isomorphic to $S_{2}\left(\Gamma_{0}(p), \mathbb{Z}\right)$ as a $\pi$-motule, as shown by Mazur [5, II, (4.6) and (6.2)]. 


\section{References}

1. P. Deligne - M. Rapoport, "Schémas de modules des courbes elliptiques," Vol. IT of the Proceedings of the International Summer School on Modular Functions, Antwerp (1972), Lecture Notes in Mathematics 349, Berlin-Heidelberg-New York, Springer 1973.

2. C. Deninger - E. NarT, Formal groups and $L$-series, Comm. Math. Helv. 65 (1990), 318-333.

3. A. GROTHENDiECK, Étude globale élémentaire de quelques classes de morphismes (EGA II), Publ. Math. IHES 8 (1961).

4. T. HONDA, On the theory of commutative formal groups, J. Math. Soc. Japan 22 (1970), 213-246.

5. B. MazUR, Modular curves and the Eisenstein ideal, Publ. Math. IHES 47 (1977), 33-186.

6. M. Raynaud, Spécialisation du foncteur de Picard, Publ. Math. IHES 38 (1970), 27-76.

7. J.P. Serre, "Groupes algébriques et corps de classes," Hermann, Paris, 1959.

Departament de Matemàtiques

Universitat Autdroma de Barcelona

08193 Bellaterra (Barcelona)

SPAIN

Rebut el 22 d'Abril de 1991 